\title{
A Tale of Two (Similar) Cities: Inferring City Similarity Through Geo-Spatial Query Log Analysis
}

\author{
Submitted for Blind Review \\ Rohan Seth, Michele Covell, Deepak Ravichandran, D. Sivakumar, Shumeet Baluja
}

\begin{abstract}
Understanding the backgrounds and interest of the people who are consuming a piece of content, such as a news story, video, or music, is vital for the content producer as well the advertisers who rely on the content to provide a channel on which to advertise. We extend traditional search-engine query log analysis, which has primarily concentrated on analyzing either single or small groups of queries or users, to examining the complete query stream of very large groups of users - the inhabitants of 13,377 cities across the United States. Query logs can be a good representation of the interests of the city's inhabitants and a useful characterization of the city itself. Further, we demonstrate how query logs can be effectively used to gather city-level statistics sufficient for providing insights into the similarities and differences between cities. Cities that are found to be similar through the use of query analysis correspond well to the similar cities as determined through other large-scale and time-consuming direct measurement studies, such as those undertaken by the Census Bureau. Extensive experiments are provided.
\end{abstract}

\section{Keywords-geographic, log analysis, large-scale similarity}

\section{InTRODUCTION: From CENSUS TO QueRY LOGS}

Understanding the backgrounds and interest of the people who are consuming a piece of content, such as a news story, video, or music, is vital for the content producer as well the advertisers who rely on the content to provide a channel on which to advertise. A variety of sources for demographic and behavioral information exist today. One of the largestscale efforts to understand people across the United States is conducted every 10 years by the US Census Bureau. This massive operation, which gathers statistics about population, ethnicity and race, is supplemented by smaller surveys, such as the American Community Survey, that gathers a variety of more in-depth information about households. Advertisers often use the high-level information gathered by these surveys to help target their ad campaigns to the most appropriate regions and cities in the US.

In contrast to the Census studies, passive studies of search engine query logs have become common since the introduction of search engines and the massive adoption of the Internet to quickly find information [9][14]. These studies provide the quantitative data to not only improve the search engine's results, but also to provide a deeper understanding of the user and the user's interests than the data collected by the Census and similar surveys.

The goal of our work is to extend techniques and data sources that have commonly been used for on-line singleuser (or small group) understanding to extremely large groups (up to millions of users) that are usually only taken on by large studies by the Census. We want to determine whether the query stream emanating from groups of users the inhabitants of 13,377 cities across the United States - is a good representation for the interests of the city's inhabitants, and therefore a useful characterization of the city itself. Figure 1 shows the geographic distribution of the queries analyzed in this study.

As a motivating example, consider whether by examining the queries emanating from cities in Silicon Valley, California could be automatically determined to be similar to other technology centers in the United States - for example in Redmond, Washington or Cambridge, Massachusetts. Beyond a city's businesses, other factors, such as weather patterns, socio-economic distributions, and ethnicities, etc., play an important role in which queries are submitted by a city's denizens.

We show that by effectively combining location information (at the city level) with search engine query logs,

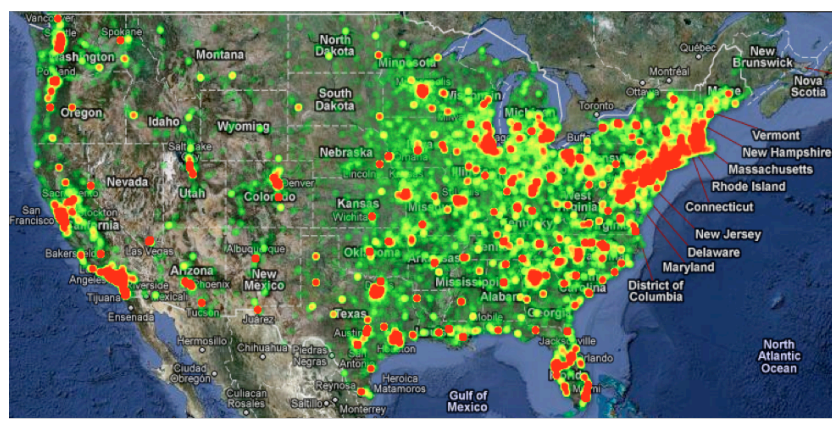

Figure 1. Geographic distribution of query samples, used in this study. 
we can ascertain the similarity of cities - even those that may not be geographically close. Finding similar cities provides a valuable signal to advertisers and content creators. Once success (or failure) is determined for the advertiser/contentcreator in one city, this analysis provides a basis for setting expectations for similar cities - thereby providing advertisers and content creators new cities to target (or avoid). Additionally, knowledge of the interests inherent in a city's population provides important information for biasing search-engine results to deliver results with a relevant local focus. It is important to note that all of the signal used in this paper can be discovered with minimal privacy concerns - individuals do not need to be identified and their individual search history need not be used.

Recently, there has been growing interest in utilizing geography as a signal for returning search engine results. Many search-engine query log analyses have examined a user's queries to better understand the user's interests and infer the user's intent [6][13][15]. The hope is that by gaining this insight, the search results returned to a user can be better tailored to the user's needs. Other studies have combined the user's IP location with the query to determine what type of content the user may be interested in [8][10] and also how to rank the search engine results in light of strong geographic signals [2][11]. Systems to efficiently combine geographic relevance with relevance as measured by more traditional information retrieval measures have been developed [3][4]. Often geographic queries do not explicitly contain location names. Nonetheless, by looking at the geographic distribution of clicks or queries, the geosensitivity of the query can be determined [16][17]. Once geo-intent is determined, language models specific to geocontent or to a particular city can be developed [15].

In order to analyze city-level query-streams, three problems must be overcome:

(1) extremely noisy query stream data: many queries
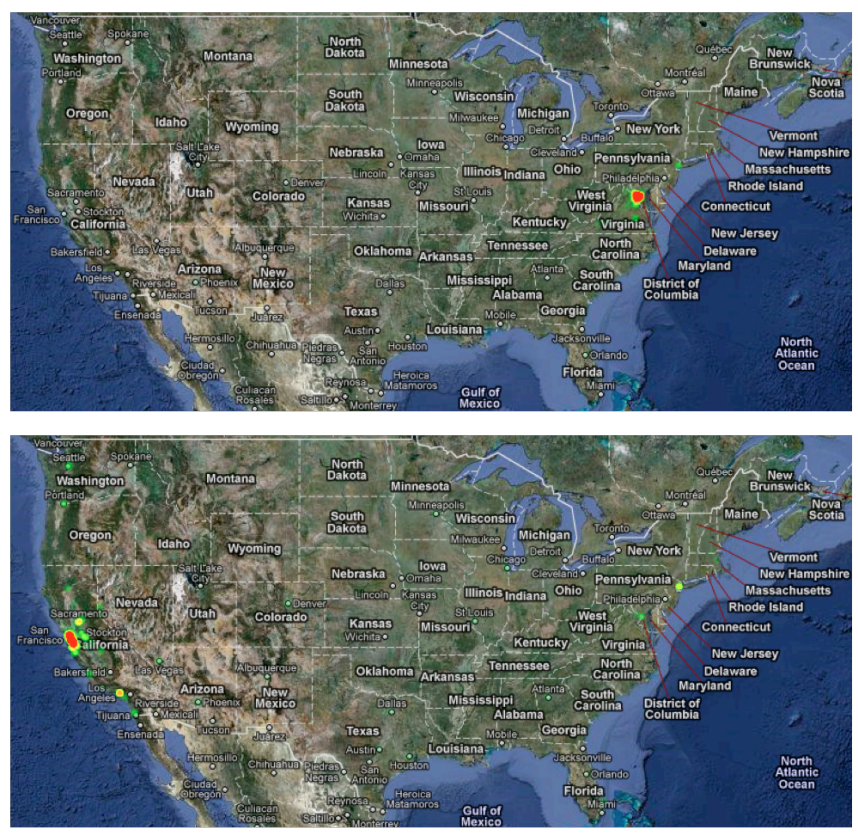

are mistakes, tests, or spam;

(2) different population sizes: computer usage and access patterns for cities make direct city comparisons difficult

(3) "regression to the mean": when looking at aggregate level statistics, at a coarse level, the diversity of populations in a city lead effectively to masking differences between cities within the differences across queries (i.e. all cities query in large amounts for "Google," "email," "Facebook," etc. and in small amounts for "chihuahua", "topaz", "babaganoush", etc.)

In the next section, we describe the creation of our ground-truth data from an independent information source as well as our basic collection of query data, to be used for our similarity measures. That section includes the method that we used to bring the location naming from these two sources into alignment, so as to allow subsequent comparison.

There are numerous manners in which search-engine query logs can be processed. In Section III, we describe two feature spaces in which to represent the basic queries: as query terms or as query concepts. In Section IV, we consider two ways to represent the feature data: by percent or by "excess score". This excess score will be the basis of many of our subsequent experiments. In Section V, we consider two different ways to infer city similarity, based on these query feature vectors: using cosine similarity or using "reverse occurrence" within the query space.

Section VI presents results from a large-scale empirical analysis of our system and a discussion of our results. The paper closes with conclusions and suggestions for future work in Sections VII and VIII.

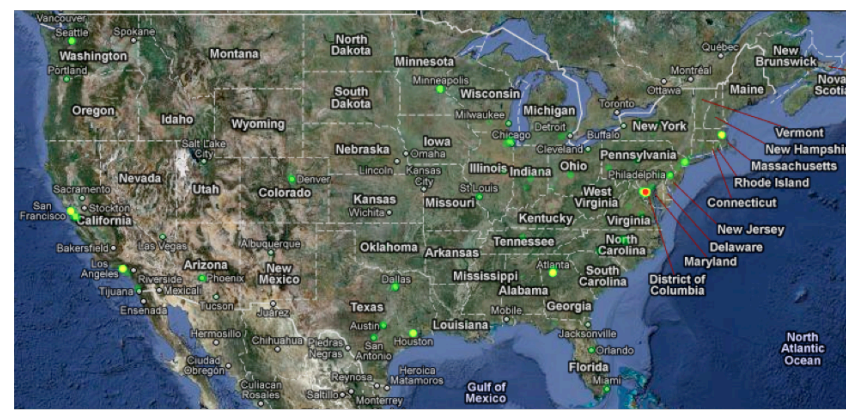

Figure 2. The query distributions for three queries that are geographically localized.

(Upper Left) "Northern Virginia Community College"

(Upper Right): "Accenture"

(Lower Left): "San Jose Mercury News" 


\section{QUERY- AND GROUND-TRUTH-DATA COLLECTION}

\section{A. Query data collection}

The analysis presented in this paper is based on seven days of logs from Google.com gathered in December, 2009. From this data, over 75 GB of summary data was extracted, based on queries originating in the United States. Based on the user's IP address that submitted the query, each query was assigned a geographic "city-level" location, using a database of IP-to-location mappings in conjunction with the Google geocoder city-level "localities" (discussed later), for a total of 13,377 distinct locations. The accuracy of the IP-togeographic mapping varies significantly depending on the location. In particular, the number, layout, and size of the internet service providers greatly affect the accuracy of the mapping. The city-level locality is at a similar accuracy level for many of the IP-address mappings. We will refer to these 13,377 localities as Cities $_{G}$.

Very simple pre-processing steps were employed to clean the query stream. First, we normalized each query by removing extraneous white spaces, special characters, and capitalizations. Second, we discarded any query that did not occur in at least 10 unique cities. No more complex heuristics were employed. Hereafter, the final set of unique queries will be referred to as $Q$.

To begin this study, we first wanted to verify our intuitions that geography is indeed a factor in query distributions [3][16][17]. Figure 2 displays a few example query distributions for terms that were highly localized: "San Jose Mercury News," "Northern Virginia Community College," and "Accenture." The maps in Figure 2 clearly show the geo-localized nature of these queries. "Northern Virginia Community College" almost exclusively appears in the northern parts of Virginia. "San Jose Mercury News" appears in San Jose and surrounding towns, a bit in Los
Angeles, and a bit in New York - places commonly associated with venture capital funding for startup technologies in Silicon Valley. Finally, "Accenture," shows that multiple geo-centers are possible and are accurately found. Many of the hot-spots for the query correspond to Accenture offices (Chicago IL; Reston VA; Atlanta GA; Denver CO) [1]. (For comparison, Figure 1 shows the distributions of data across all the queries gathered for this study.)

In contrast to the geographically localized queries in Figure 2, the geographic distribution of other queries, for example those of a more general interest, exhibit far less geographical coherence. A few such examples, "email", "Google", and "Christmas," are shown in Figure 3.

\section{B. Ground-truth data collection}

There is no definitive measure of city-to-city similarity. Often, simple measures such as distribution of income levels, ethnicity, or education level are used. However, each of these attributes only captures a specific, small aspect of a city. Rather than limiting our analysis to a single dimension or risking introducing potential sources of bias in our analysis by hand-selecting a single or small set of attributes as our target, we took a more comprehensive and automated approach.

We gathered over 750 city-to-city similarity lists, based on information in the census-related surveys. These lists only contained "the top 101" cities (and counties) in the United States, in each of the 750 categories. List categories ranged from the expected (population size, percentage of population with advanced degrees, average income level, etc.) to the obscure (largest percentage of males working as cashiers, smallest percentage of divorced people, etc.). Each one of these lists was used as a component in our ground-truth calculations. To create our ground truth
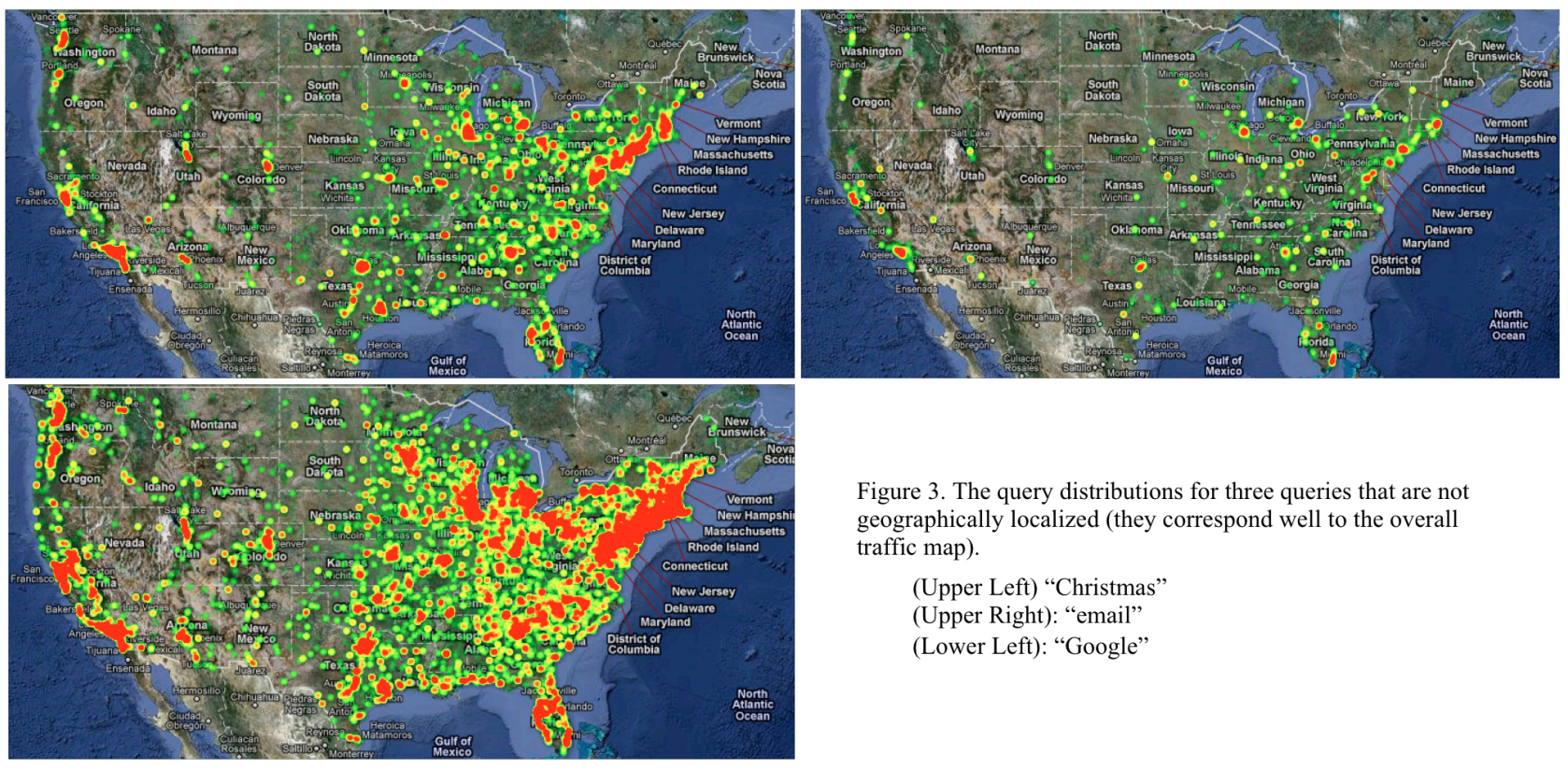

Figure 3 . The query distributions for three queries that are not geographically localized (they correspond well to the overall traffic map).

$$
\begin{aligned}
& \text { (Upper Left) “Christmas" } \\
& \text { (Upper Right): "email” } \\
& \text { (Lower Left): "Google" }
\end{aligned}
$$


similarities, we simply count, for all pairs of cities, the number of lists on which they co-occur. The end result is a "city-to-city" association list that contains pairs of 12,873 unique cities and counties. The highest-co-occurrence of any two cities is 30. A wide range of cities is included in these 12,873: for example, ranging in population from 9.5 million (Los Angeles County CA) to 95 (Indian Beach NC). This final list has the advantage of measuring similarity across a large set of dimensions, and avoids testing biases that could have otherwise been introduced by manually choosing the most interesting criteria.

It is important to remember that this namespace of 12,873 cities and counties have been generated by a completely separate process than the logs analysis that generated the 13,377 Cities $_{G}$ set. The set of 12,873 location names are simply what was present on the 750 "top 101 " lists that we used, derived the Census data. The 13,377 Cities $_{G}$ set are based on the geo-coding of the IP addresses we saw in the query $\operatorname{logs}$ that we analyzed. As such the names generally will not match. We address this difficulty in the next subsection.

While all 12,873 of city-to-city locations have associations with 100 other cities (since each list has 101 entries), many of them have only that one list as the way they are characterized in the city-to-city data. This weak information will be very biased by which list that city was listed on, which is exactly what we are trying to avoid by using 750 lists. To avoid this problem, we will only consider as target cities for evaluation, those cities that have at least one cohort city that shared lists with them at least 3 times. This reduces the list of "target cities" down to 8123 city-to-city locations. We continue to use the full list of 12,873 as potential partners to each target city, using the cooccurrence to weight the associations and give more emphasis to city pairs that occur more frequently

\section{Location alignment and target city evalutaion}

As noted above, the ground-truth and query-analysis results are built on two separate namespaces. To try to bring the two together, we passed each city-to-city name through a geo-coder supplied by http://maps.google.com. Each location was translated into latitude and longitude coordinates. We then reverse geo-coded each of these 12,873 latitude/longitude coordinates, mapping each to the city-level "localities" used by Google.com. These forwardreverse geo-coding operations resulted in many-to-one assignments from the 12,873 city-to-city lists to Google localities. While all of these mapped into the Cities $_{G}$ set (for which we have query-log data), the mapping was such that they mapped onto only 8093 Google localities. Many-to-one mappings typically occurred when counties from the city-tocity lists mapped to the same locality as one of the alsoreferenced cities within the county. In addition, we also saw within-city neighborhood names from the city-to-city lists mapping onto the same city-level locality.
In order to describe how we handle this ambiguity, we first consider how we will eventually evaluate the quality of our similarity results. In Section VI, we provide a variety of analysis tools. In all cases, we cycle through a list of target cities, one at a time, measuring for each the qualities of our similarity results (given by logs analysis) compared to what was given by the ground truth (given by the city-to-city lists).

So, we define the "target city" as the city-to-city location to which we want to measure or rank all the known cities. In order to decide which Google locality to use as the target city, we simply use the forward/reverse geo-code mapping described above. This gives us a pair of vectors to compare: one from the city-to-city namespace, containing sparse mappings within a set of 12,872 other city-to-city names and the other from the Google locality namespace, giving us a dense mapping to 8093 other Google localities that are their geo-coding-based "partners". Note that, based on the list occurrence requirements mentioned in the previous subsection, there are 8123 distinct target cities, which then map onto only 4478 Google localities. The full 8123-target set is distinct since the target city data is evaluated as a pair and the city-to-city sparse mappings will be different for each of the 8123 names, even when the query-analysis mappings are repeated by the many-to-one nature of the association.

For each of these target cases, we now need to decide how to compare a sparse association mapping into the 12,872 city-to-city namespace with a dense mapping into the 8093 Google query-stream localities. For many Google localities, there is only one city-to-city name with a default mapping onto that Google locality name. We fix these mappings as our first step. For the remaining Google localities, where the association to a city-to-city name is ambiguous, we use an optimistic mapping onto city-to-city names that have not already been used. The greedy mapping is done starting from the most strongly associated Google locality (that is in the ambiguous set) and picking (from the city-to-city set within the $30 \mathrm{~km}$ radius) the most strongly associated city-to-city name that has not already been used.

The mapping is used in all evaluations, including the baseline orderings by geographic distance, total population, and population difference, discussed in Section VI. As such, the evaluations done relative to those measures provide an accurate indication of the quality of our similarity predictions, even though our greedy mapping will bring our results into closer alignment with the ground-truth data than might be warranted - as long as we only pay attention to the relative performance, compared to the these alternatives which have the same optimistic advantages, we will not overstate our results.

\section{Feature Space for Processing Query LogS}

One of the difficulties in comparing queries, even after standard normalization steps are taken, is that queries that 
may initially appear to be far apart, in terms of spelling and edit distance, can represent the same concept. For example, the terms "auto" and "cars" are often used interchangeably, as are "coke" and "pop" or "mobile" and "phone." To treat these sets of queries as similar, we replace each query with a concept cluster.

Concept clusters are based on a large-scale Bayesian network model of text, as detailed in [5][7]. Datta describes the creation of PHIL (probabilistic hierarchical inferential learner). Although a full explanation of the PHIL system is beyond the scope of this paper, a cursory overview is provided here. PHIL is a top-down weighted directed acyclic graph in which the top node represents "all concepts" and the leaf nodes represent individual words or compound-word tokens. The intermediate nodes, which can be learned through word co-occurrence statistics over large text corpora, are created automatically. The intermediate nodes contain many conceptually similar words. PHIL was originally used as a generative model of text. For our purposes, each query is used as input to the system, and the intermediate nodes that are most highly activated are assigned to the query. Similar concept queries will activate similar nodes. Interestingly, this system not only helps account for different words often used to represent the same concept (e.g., autos/cars) but it also helps to automatically correct for common spelling mistakes: the same nodes will be activated for common variations of the same word.

In Section VI, we will empirically compare the use of terms and PHIL clusters as the underlying features for our system.

\section{Query Signatures OF Cities}

For each city, we need to compute a signature vector that will be used to compare with the signature of other cities. In addition to the choice between query terms and query concepts, described in the previous section, we must consider purely descriptive versus discriminative signature vectors.

The simplest signature is a (descriptive) histogram of the queries that originated from the city. This has a straightforward computation: for each unique query (whether term or concept), $q$, that occurs from any city in our collected set, $Q$, we count how many times the query originated from a city, $c$. Each city is assigned a vector of integers, of length $|Q|$. Many smaller cities will have numerous zero entries; these indicate that the query never occurred in the city during the sampling time. To address the problem of the wide range of query volumes across different cities, the histogram is normalized so that each query's entry reflects the percentage of the total query volume the query accounts for.

A limitation of this simple histogram approach is seen in many descriptive signatures: it may place emphasis on noninteresting portions of the query-space. For example, we expect that a common query such as 'pizza' will occur far more often than a query such as 'babaganoush,' or that 'blueray' will be far more numerous than 'cassette tape.' The fact that these relations repeatedly hold in almost every city is neither interesting nor surprising when trying to find how cities differ. Rather, the converse is true: when these relations do not hold is when the data is interesting.

To draw attention to the surprise features of a city, we use a simple discriminative scheme that is analogous in spirit to the family of TF-IDF [12] procedures commonly employed in information retrieval tasks. We call the measure an excess score. Intuitively, the goal of an excess score is to determine how much above (or below) each query was from the expected. We determine how much we expected to see a query by treating the entire set of queries from all cities (Cities $_{G}$ ) as a single city, and computing the distributions of queries in this aggregated set. Specifically, it is computed as follows. First we compute the expected volume of the query averaged across all cities. This percentage becomes our expectation for that query:

expectedPercentage $_{\text {query }}=$

$$
\sum_{c \in \text { Cities }_{G}} \text { Volume }_{c, q u e r y} / \sum_{c \in \text { Cities }_{G}} \sum_{q \in Q} \text { Volume }_{c, q}
$$

Using the expected percentage, we then compute how the query volume in a city differed from the percentage we expected from that city, given its total volume:

$$
\text { excess }_{\text {query|city }}=\frac{\text { Volume }_{\text {city }, q u e r y}}{\sum_{q \in Q} \text { Volume }_{\text {city }, q}}-\text { expectedPercentage }_{\text {query }}
$$

Four points should be made about the excess score. First, as shown above, it calculated the "surprise factor" of each query per city - it does not represent the volume directly. Alternatively, we could have simply modified the calculation to find the excess cities for each query - i.e., for each query, find which cities had a higher (or lower) than expected occurrence of that query. First we compute the expected query volume of a city, averaged across all queries, and then we estimate that city's expected percent contribution to the total number of queries examined in the study.

$$
\begin{gathered}
\sum_{q \in Q}^{\text {expectedPercentage }_{\text {city }}}= \\
\text { excess }_{\text {citylquery }}=\frac{\text { Volume }_{\text {city }, q u e r y}}{\sum_{c \in \text { Cities }_{G}} \sum_{q \in Q} \text { Volume }_{c, q}}-\text { expectedPercentage }_{\text {city }}
\end{gathered}
$$

Second, it should be noted that the excess scores can be negative. Intuitively, for the cities that have a negative excess score for a query, in that city, the query may have occurred less than expected.

Third, the excess score for the queries for any city should sum to 0.0 . The fact that some query occurred more times than expected when looking at the total volume of queries from the city implies that some other query (or set of queries) must have occurred less than expected when looking at that same volume of queries.

Fourth, for simplicity, we calculated the expectation as the 'global expectation,' using all the cities in Cities $_{G}$. An alternative would be to compute more specific expectations, 
such as using only the nearest $n$-cities, or dividing the U.S. into quadrants, etc.

In the experiments presented in Section VI, we will use the excess scores as well as the simple percentage-histogram approach in determining city similarity.

\section{INFERRING SIMILARITY FROM QUERY VECTORS}

Given the excess metrics as well as the simple percentage based signatures, described in the previous sections, we need to decide how to compute the distance between two cities. We consider two alternative methods. The first method is the standard cosine similarity, which can be used with the excess vectors as well as the percentage based vectors. The cosine similarity between two cities A and B is defined as:

$$
\text { Similarity }=\cos (\theta)=\frac{A \cdot B}{\|A\|\|B\|}
$$

A second similarity metric is based on computing the excess scores for a city given a query (excess citylquery $_{\text {). For }}$ this calculation, we examined every query in $Q$. For each query, $q$, cities were assigned their excess score and the list was sorted. This sorted list represented which cities had the highest (positive) "surprise factor" for $q$, taking into account their expected total volume. From each list, all cities that co-occurred within the top-10 query excess scores had their similarity score incremented. Intuitively, this measures how many times each pair of cities coincided on having more than expected query volumes for a particular query. We refer to this similarity measure as "reverse-occurrence voting".

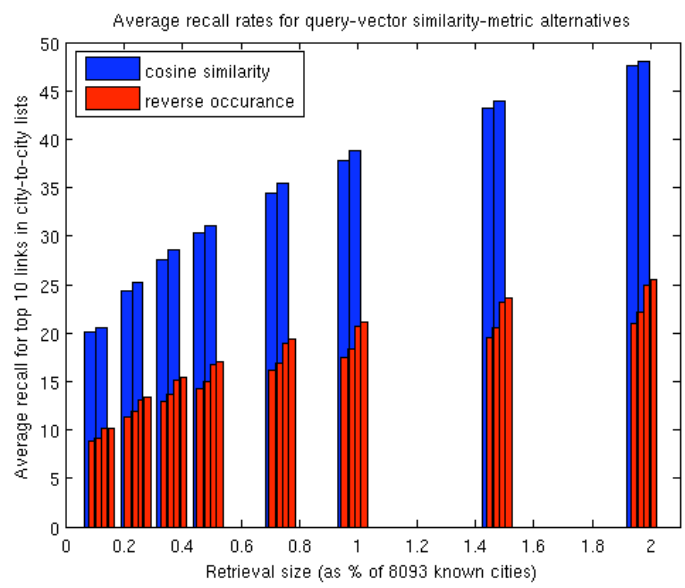

Figure 4 Using cosine similarity vs. reverse-occurrence voting. The average retrieval rates for the top-10 matches for each city when retrieving N\% ( $x$-axis) of the cities - ordered by similarity - either through cosine distance or reverse occurrence, is shown. For example, when cosine distance is used to measure city similarity and $1.5 \%$ of the cities are retrieved, approximately $43 \%$ of the top- 10 most similar cities are included. When reverse occurrence similarity is used, only $20-24 \%$ of the top- 10 closest cities are included. This significantly lower performance is true whether we use query terms or PHIL clusters and whether we use excess or percentage features.
The problem with reverse-occurrence voting is that there is no guarantee that every city will be voted for, even once, by the query set that was seen. Even with more than 12 million distinct query terms, some cities (typically small ones) never rise to the top 10 of even a single query list. Nearly one fourth of our target cities suffered from this anonymity. As can be seen in Figure 4, even those cities that did make it to some type of characterization were more poorly described by the reverse-occurrence voting than by the cosine similarity.

In Figure 4, we show a comparison of the performance of cosine similarity and reverse-occurrence voting, where both measures were taken over only the target cities that were not anonymous under reverse-occurrence voting (just over 6000 city-to-city names). The figure shows the performance for all four combinations from Sections III and IV: query terms or PHIL clusters with excess or percentage scores. The graph shows how many of the top-10 most strongly connected cities were retrieved using cosine similarity versus reverse-occurrence, according to the retrieval-set size. The retrieval set is taken from the ordering of most-toleast similar cities given by each of the two alternate measures. In Figure 4, the x-axis is the size of the retrieval set (as a percentage of the full 8093 possibilities) and the yaxis is the average recall rate for these top city-to-city localities, averaged across the non-anonymous target cities. Note that the average retrieval using cosine similarity is, for this one graph, also averaged on this reduced set of cities that was not anonymous under reverse-occurrence voting.

Not only does reverse-occurrence voting leave many cities completely uncharacterized, but it also provides only about half of the recall rate, compared to cosine similarity, for the same size retrieval set. For this reason, the remainder of this paper uses cosine similarity on the query vectors (query terms or PHIL clusters in excess scores or percentages) to determine city similarities.

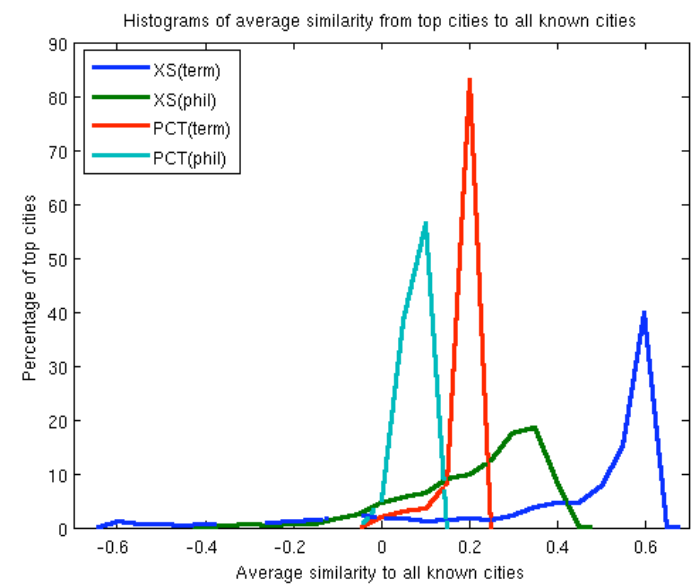

Figure 5 Histogram of the average similarity, from each target city to all known cities. The strongly positive biases show that the query vectors, whether terms or clusters, percent or excess, vary across our localities in strongly correlated ways. 


\section{EXPERIMENTS}

As discussed in Section II-C, our method for mapping cityto-city names onto Google locality names will tend to bias our evaluations towards aligning our similarity lists to be more in agreement with the city-to-city lists than would have been provided without these naming ambiguities. As a result of this systematic bias, we need to separately measure the improvement provided the optimistic name remapping and remove it from our evaluation results. Throughout this section, we match the bias-removal method to the summary statistic being shown.

One summary statistic that we provide is the weighted connected similarity: measured from each target city to all of the cities with which it co-occurred in the city-to-city lists, it is the cosine similarity, weighted by the shared-list count. We correct this simple statistic using two factors. The first corrects for the similarity that each target city shows to all known cities. The second corrects (on average) for the bias introduced by the optimistic namespace mapping. By correcting for both, we can interpret a zero offset similarity as being equivalent to random selection of neighbor cities.

Figure 5 shows the histograms of the first correction factor: for each target city, what similarity its query vector showed, on average, with the query vectors of all of the other known cities. There is a very strong positive similarity seen in all of our query-based similarity measures for our locality set. This strong positive average similarity across all of our known localities is easy to understand for the percentage measures: all of the values for the percentage features will be positive, since all percentages are positive, so measuring cosine similarity on these vectors will always give a positive result. The even stronger positive similarity seen in the excess measures shows that directions of variations in the underlying percentages are also strongly aligned: even taking a differential measure of the per-location query vectors does not scatter these vectors uniformly across the vector space. High positive average similarities limit the offset similarity that we can achieve: that is, the weighted similarity of the linked cities relative to (or offset by) this all-city average. Even so, high positive average similarities suggest that our excess scores are not measuring noise only: our query vectors do contain a lot of related entries that should show synchronized variation, which will remain even after taking the excess score.

The second correction factor is a further offset to the similarity score to, on average, reduce the effect of the optimistic namespace mapping that we are doing. For each type of query vector (term or PHIL, excess or percentage), we looked at the scores of all the cities available for any of the ambiguous namespace mappings that were within the 30 $\mathrm{km}$ radius of an ambiguous Google locality. Within each of these $30 \mathrm{~km}$ sets, we measured the standard deviation in the similarity score and then took the average of all these standard deviations, across all of the sets. This gives us an average measure of how optimistic our namespace mapping will tend to be, according to the query vector type. Since these localized standard deviations are not the same for the different query-vector representations, we need to reduce the offset similarity scores by these representation-dependent amounts before comparison. For the four different query representations, these corrections reduced our reported offset similarity: for percentage query terms and PHIL clusters, by less than 0.01; for excess PHIL clusters, by 0.06 ; and for excess query terms, by 0.13 . Our histograms, shown in Figure 6 , have had their offset similarity scores reduced by these amounts before plotting.

Based on Figure 6 (left) and (center), the excess score is more accurate than the percentage scores in finding similar cities. The excess scores are nearly always doing better than simple random association, as shown by the excess bars (yellow) being $100 \%$ of the target cities above the zero offset-similarity score. In contrast, only $58 \%$ and $60 \%$ of the target cities are doing as well or better than random, using percent PHIL clusters and percent query terms, respectively. Based on this weak result for the percentage terms, we do not consider it further.

Figure 6 (right) suggests that the excess-PHIL vector is giving a stronger similarity signal than are the excess-term vector: the excess-PHIL vector has nearly $50 \%$ of the target cities at or above an offset similarity score of 0.3 , while the excess-term vector has only $10 \%$ of the target cities at or
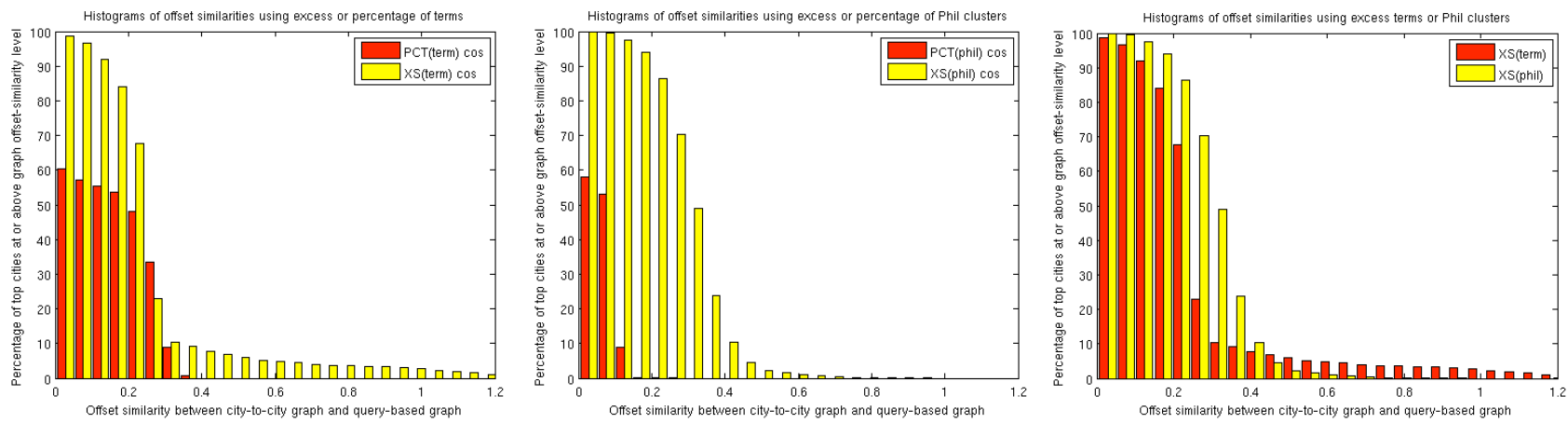

Figure 6 Offset similarity histograms. These histograms show the weighted-average similarity from each target city to their city-to-city connected localities, offset by the average similarity of that target city to all known localities and further reduced by the average ambiguous-neighborhood standard deviation for the selected representation. The comparisons in the left and center graphs show that the excess-based representations do better than the corresponding PHIL-based representations. The comparison on the right shows that, by this measure, the excess-PHIL representation does better than the excess-term representation. 
above that level. However, since we have not adjusted these two offset-similarity populations for differences in dynamic range, we cannot draw any substantive conclusion from this comparison. Normalizing by the standard deviation will not help, since the excess-term vector similarities form a superGaussian (heavy-tailed) distribution while the excess-PHIL vector similarities give a sub-Gaussian (weak-tailed) distribution.

In order to further evaluate the relative performance of excess-PHIL and excess-term vectors, we look at the detailed recall behaviors of the two approaches. We do this, for each target city, by looking for 10 cities that were most strongly associated with that target city in the city-to-city lists, within the list of known cities, as ordered by the query-analysis similarity. For example, for Cambridge MA, we would look for Berkeley CA, Stanford CA, Brookline MA, Somerville MA, Boston MA, Albany CA, New York NY, Hanover NH, Ithaca NY, and Bethesda MD, based on the city-to-city lists.
We would then count how many of these 10 cities were among the top $0.1 \%$ (10 of 8096$), 0.2 \%$ (20), $0.4 \%$ (30), $0.5 \%$ (40), $0.7 \%$ (60), $1 \%$ (80), $1.5 \%$ (120), and $2 \%(160)$. Continuing with our Cambridge MA example, using the excess-term vectors to order our retrieval results, we see four of these top cities in the first 10 results (namely, Boston, Brookline, New York, and Bethesda), 5 cities in our first 20 results (adding in Stanford), 6 cities in our first 30 results (adding in Somerville), 7 cities in our first 40 results (adding in Ithaca), 8 cities in our first 60 results (adding in Hanover), and 9 cities in our first 80 results (adding in Berkeley). The tenth city (Albany) does not occur in our retrieval results until position 212, so it is not included in our reported results.

When we do this more detailed evaluation of the mostsimilar recall rates, the differences in recall rates between excess-PHIL and excess-term are not significant. ExcessPHIL processing gives, on average, $1.5 \%$ to $2 \%$ better recall
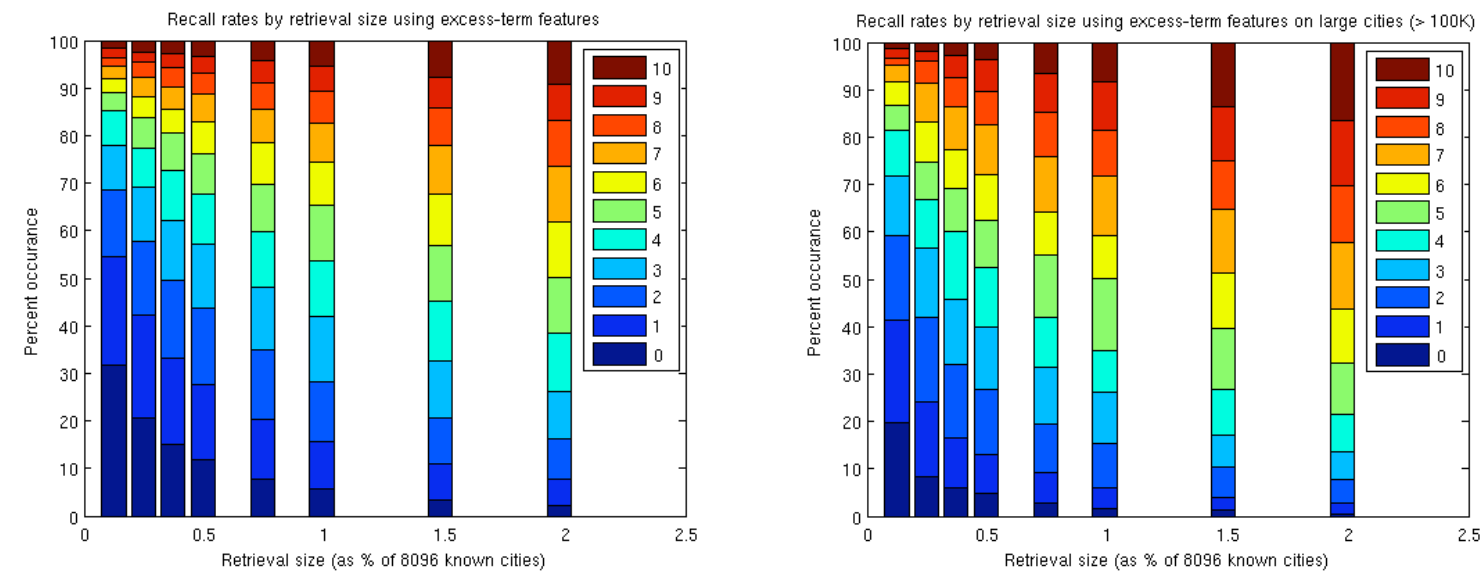

Figure 7 Recall rates, by retrieval-set size, using excess-term features. Each bar in the above graphs show the recall distribution, across target cities, in looking to find the top 10 city-to-city associations in the retrieval result provided by excess-term similarity sorting. The colors correspond to different recall rates, from finding all 10 cities (top, red) to finding none (bottom, blue-black). The x axis indicates the retrieval-set size, as a percentage of the full set of 8096 known cities, ranging from $0.1 \%$ up to $2 \%$. The left graph shows the results across all target cities. The right graph shows the results on large cities ( $>100 \mathrm{~K}$ population).
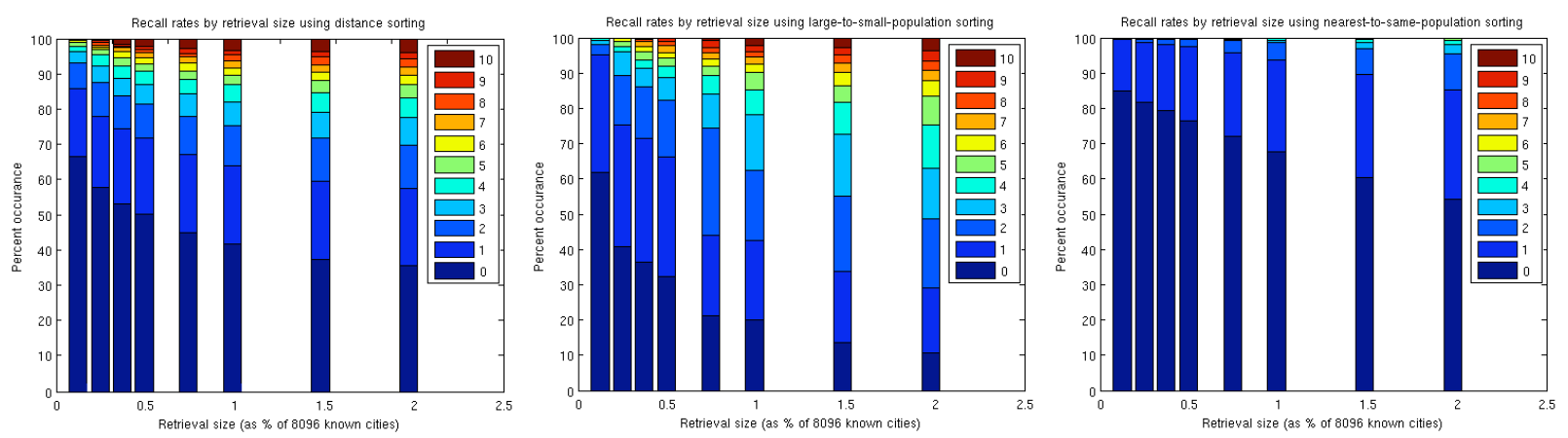

Figure 8 Recall rates, by retrieval-set size, using various baseline methods, for comparison. These graphs provide baseline performance levels for the recall according to retrieval-set size. The graph structure is the same as in Figure 7, except for the method used to select the retrieval set. On the left, we use geographic distance to sort the known cities. At center, we use the city population size (from largest to smallest) to sort the known cities. On the right, we use the difference in population size compared to the target-city size (from closest in size to most different). For all three of these graphs, we used the same optimistic namespace mapping as was described in Section II-c. 
than excess-term across the considered retrieval sizes (where recall of all 10 top cities corresponds to $100 \%$ recall). Excess-term does better than excess-PHIL on large cities while excess-PHIL does better on mid-sized and small cities. However, the standard deviation in these recall differences are on the order of $12 \%$ to $15 \%$ and none of these differences even approach statistical significance. Based on this lack of significance, we only show the results of the excess-term vector for the remainder of this paper.

Figure 7 shows the details of our recall results, across retrieval-set size, for all target cities. The $\mathrm{x}$ axis is the retrieval set size. The different colors in stacked bar graphs at each $\mathrm{x}$ position show how many of the sought-after 10 cities were seen at that retrieval size and the size of each colored section show what percentage of the target-city set got that level of recall, at that retrieval size. Returning to our Cambridge example, this city would be included in the " 4 " color region, at the $0.1 \%$ retrieval size (with higher recall than $79 \%$ of the other target cities at that retrieval size); in the " 5 " color region, at the $0.2 \%$ retrieval size (better than $78 \%$ of the target cities); in the " 6 " color region at $0.4 \%$ recall (better than $81 \%$ of the target cities); in the " 7 " color region at $0.5 \%$ recall; and so on.

We again need to provide a baseline result against which to compare. While it is tempting to compare to random sampling without replacement, that comparison is too optimistic due to our namespace mapping process. Instead, in Figure 8, we show three baseline orderings: by distance, by population size, and by population difference. For our distance ordering, for each target city, we sort all known cities according to their distance from the target. This is based on the hypothesis that places that are near each other are most similar. After we have done this initial sort, we repeat the same namespace mapping as was described in Section II-C, so the distance mapping can receive the same boost to its recall results. Our second baseline ordering is by population size. We sort all known cities once, according to their reported population size (largest to smallest). We use this single ordering as a starting point for all target cities but allow it to be modified for the ambiguous names, again using the mapping described in Section II-C. Our final baseline ordering is by population difference. For each target city, we sort all known cities, according to their (absolute) population difference compared to that target, so that cities that are of similar size to the target will occur early in the list and cities that are much larger or smaller will occur late in the list. We again use the namespace mapping from Section II-C.

As can be seen by comparing Figures 7 and 8, the recall results for the excess-term-based sorting are much higher than what would be provided by any of our simple baseline methods. Since the baseline methods enjoy the same optimistic namespace mapping as our excess-term results, our results are not due to that mapping. In Figure 7, we can also see an improvement from the average target-city recall performance to that of the largest target-city recall.

To gain a better understanding of our derived similarities, we look at the results from three well-known, mid-sized locations: Cambridge MA, Austin TX, and Redmond WA. Cambridge is the home of two prestigious universities (MIT and Harvard), as well as being a center for high-tech startups and defense contracting. When we look at the top associated cities, the first three associated localities share some of these characteristics but most likely are listed due to geographic location: Boston MA, Brookline MA, and Waltham MA are all very close to Cambridge. In addition to those nearby cities, we see associations that are best explained by character: Palo Alto CA has a high quality university (Stanford), as well as many high-tech companies, both startup (Facebook, VMware, etc) and established (HewlettPackard, Xerox PARC, Fuji-Xerox Research). There is a cluster of associated cities from the defense-contractor centers: specifically, Bethesda VA, Arlington VA, and Fort Myer VA. The association with Irvine CA is may be due to its mix of academics (UC Irvine) and high tech (Broadcom). Bellevue WA is also closely associated, probably since it is near Microsoft.

Similar types of associations can be seen for Redmond WA (Microsoft Headquarters). Two nearby communities are most strongly associated: Seattle WA and Bellevue WA. There is a cluster of cities from the Boston area, which share the high-tech bias: namely, Cambridge MA, Waltham MA (with many high-tech start ups), and Lexington MA (with MIT Lincoln Labs). The largest group of associated cities for Redmond is from the Silicon Valley area: Sunnyvale CA, San Jose CA, Mountain View CA, and San Francisco CA. In addition, Buffalo NY (with SUNY) and Oakland CA (with Berkeley) are associated with Redmond, probably based on their engineering schools.

Austin TX shares many of the same characteristics and association but, in addition, shows a bias towards government centers, such as Frankfurt KY (state capital) and Washington DC. The high-tech associations for Austin are less obvious than those of Cambridge and Redmond but probably account for its association with Norcross GA (Megatrends Corp) and San Bruno CA (YouTube). Other associations to Austin included: geographic location (Mc Neil TX), defense contracting (Ellicott City MD, Crofton MD), and academics (Atlanta GA, for Georgia Tech as well as for many high-tech startups).

\section{CONCLUSIONS}

In this paper, we have employed techniques that have previously been used to examine either single or small sets of users and extended the procedures to analyze the populations of more than 13 thousand cities across the U.S. Despite the diversity of people in cities, we are able to find signals in the aggregate query streams emanating from the cities and use them to determine similar cities that are not necessarily geographically close.

The most important signal we used for our analysis, the excess score, was both simple and easy to compute. Intuitively, it measures the 'surprise' in the volume of a particular query. This measure helps us overcome two important difficulties with the data. First, every city has many queries for common terms; instead of simply eliminating these common terms (as is often done with stoplist type approaches), the effect of these terms is reduced 
unless there is a reason to pay attention to the terms (i.e. they occur either more or less than expected in a city). Second, the excess measure also provides a simple basis with which to normalize for the query volume of a city. This was crucial, considering the wide range of population sizes and search engine usage in the cities examined.

The results obtained by our system perform well even with small retrieval sets. With only retrieving 160 cities ( $2 \%$ of the known cities), on average, we find 6 cities from the top-10 closest cities (as measured by the census-databased city-to-city dataset). Other, intuitive heuristics, such as geographically closest 160 cities, or 160 cities with the closest sized population, performed significantly worse.

\section{FUTURE DIRECTIONS}

There are a number of future directions for exploration. In terms of the algorithms, one of the first experiments to conduct is with weighted excess metrics. Currently, each term is normalized such that its contribution is proportional to its deviation from its expected volume. However, some terms may be more important than others - for example, if a term that was expected to account for a large percentage of a the query traffic didn't (i.e. there was only $1 / 10^{\text {th }}$ the number of expected queries of a popular term like "Twitter"), that may be more telling about the population than the drop of a less popular term (i.e. $1 / 10^{\text {th }}$ the volume of the query "Pinto muffler"). One simple method to incorporate an importance weighting is to multiply the excess score with the query's expected percentage of traffic. Experiments need to be conducted to see whether such a weighting translates into improved performance.

In this paper, we attempted to find similar cities by looking at their query distributions. Alternatively, we could also address the task of finding related queries by looking at their excess distributions across cities. Even more interesting would be to combine the two analyses, so as to allow specialization of our city-similarity lists to coherent clusters of concept variations, such as weather or academic emphasis, without manual selection of the query dimensions.

In this study, we created a ground-truth set from lists of similar cities on the web. In the future, we will compare our performance directly to Census data. By using the Census data, and creating multiple association lists from various segments of their data (for example the most similar cities based on household income, job type, ethnicity, etc), we can further understand which segments of their data corresponds closest to the similarity that is found through analyzing query logs.

Perhaps most important to large scale adoption of this work, we need to measure how the similar-city lists found here correlate with the success and failure of content and advertising campaigns that have been launched in multiple cities. Understanding this, first through historical log analysis and then through controlled trials, will be an important step towards understanding the extent to which the city-similarities can be used for helping content-creators and advertisers.

\section{REFERENCES}

[1] Accenture, Inc. U.S. Office Locations http://www.accenture.com/ Countries/USA/About_Accenture/USOffices.htm

[2] L. Andrade and M.J. Silva (2006). "Relevance Ranking for Geographic IR." In Proc. ACM SIGIR Workshop on Geographical Information Retrieval (Seattle WA).

[3] L. Backstrom, J. Kleinberg, R. Kumar, and J. Novak (2008). "Spatial Variation in Search Engine Queries." In Proc. International Conference on World Wide Web (Beijing, China), pp. 357-366.

[4] Y. Chen, T. Suel, and A. Markowetz (2006). "Efficient Query Processing in Geographic Web Search Engines." In Proc. ACM SIGMOD International Conference on Management of Data (Chicago, IL), pp. 277-288.

[5] R. Datta (2005) "PHIL: The Probabilistic Hierarchical Inferential Learner," presented at the $10^{\text {th }}$ Annual Bay Area Discrete Mathematics Day. http://math.berkeley.edu/ $\sim$ datta/philtalk.pdf

[6] Q. Gan, J. Attenberg, A. Markowetz, and T. Suel (2008). "Analysis of Geographic Queries in a Search Engine Log." In Proc. ACM International Workshop on Location and the Web (Beijing, China), pp. 49-56.

[7] G. Harik, N. Shazeer (2004) "Method and Apparatus for Learning a Probabilistic Generative Model for Text," U.S. Patent 7231393.

[8] A. Hassan, R. Jones, and F. Diaz, (2009). “A Case Study of using Geographic Cues to Predict Query News Intent." In Proc. ACM SIGSPATIAL International Conference on Advances in Geographic information Systems (Seattle, Washington), pp. 33-41.

[9] B.J. Jansen and A. Spink (2006). "How are We Searching the World Wide Web? A Comparison of Nine Search Engine Transaction Logs." Information Processing and Management 42 (1): 248-263.

[10] R. Jones, W.V. Zhang, B. Rey, P. Jhala, and E. Stipp (2008). "Geographic Intention and Modification in Web Search," Int. J. Geographical Information Science. 22 (3): 229-246.

[11] R. Jones, A. Hassan, and F. Diaz, (2008). "Geographic Features in Web Search Retrieval." In Proc. International Workshop on Geographic Information Retrieval (Napa Valley, CA), pp. 57-58.

[12] G. Salton and M.J. McGill (1983). Introduction to Modern Information Retrieval. McGraw-Hill. ISBN 0070544840.

[13] T. Sanderson and J. Kohler (2004). "Analyzing Geographic Queries." In Proc. ACM SIGIR Workshop on Geographic Information Retrieval (Sheffield, UK).

[14] C. Silverstein, H. Marais, M. Henzinger, and M. Moricz (1999). "Analysis of a Very Large Web Search Engine Query Log." SIGIR Forum 33 (1): 6-12.

[15] X. Yi, H. Raghavan, and C. Leggetter (2009). "Discovering Users' Specific Geo Intention in Web Search." In Proc. International Conference on World Wide Web (Madrid, Spain), pp. 481-490.

[16] Z. Zhuang, C. Brunk, and C.L. Giles (2008). "Modeling and Visualizing Geo-Sensitive Queries based on User Clicks." In Proc. ACM International Workshop on Location and the Web (Beijing, China), pp. 73-76.

[17] Z. Zhuang, C. Brunk, P. Mitra, and C.L. Giles (2008). "Towards Click-Based Models of Geographic Interests in Web Search." In Proc. IEEE/WIC/ACM International Conference on Web Intelligence and Intelligent Agent Technology (Sydney, Australia), pp. 293-299. 